\title{
Editorial
}

\section{Anaesthesia for the Stroke Patients - New Challenges for Anaesthesiologist}

Neurosurgical anaesthesia focuses on stroke patients undergoing craniotomy as an urgent nature with life threatening conditions. In developed countries coronary heart disease and stroke are the first and second leading cause of death respectively among adult men and women. Cardiovascular diseases killed 17.5 million people in 2012 that is 3 in every 10 deaths. Of these, 7.4 million people died of ischemic heart disease and 6.7 million from stroke. However, the burden of stroke in developing countries has been increasing significantly. Twice as many deaths from stroke occur in developing countries as in developed countries. Overall in developing countries stroke ranks second or third in disease burden. By 2020 stroke is thought to be the leading cause of death in developing countries. Deaths rates from stroke for people of $<65$ years have fallen by $23 \%$ in the last 10 years in developed countries but in developing country still it is remaining higher. In the last 10 years a significant increase in the life expectancy has occurred in developing countries like Bangladesh. So there is increase in the number \& incidence of cerebrovascular diseases in Bangladesh. The need for emergency neuro anesthesia for the stroke patients are gradually increasing both for intracranial neurosurgical \& neuroendovascular procedures. The mortality in patients requiring emergency neurosurgical procedures is still quite high.

Stroke is the third leading cause of death in Bangladesh (8.57\%). Among the strokes $70-80 \%$ are ischemic \& 20-30\% are hemorrhagic. Ischemic strokes (thrombotic strokes and embolic strokes) are mostly managed conservatively by removing the obstruction which help to restore blood flow in the brain. Carotid endarterctomy, stenting of the cervical and intracranial vessels or decompression craniotomy are the mostly used surgical and non surgical maneuvers in the management of ischaemic stroke patients and these maneuvers may help in reducing recurrent stroke in some cases or reduce intracranial pressure due to edema.
2-5\% patients needs anaesthesia support during these maneuvers.

A hemorrhagic stroke(intracerebral and subarachnoid) can be caused from hypertension, rupture of an aneurysm or vascular malformation, or as a complication of anticoagulation medications.

$10-20 \%$ of hemorrhagic stroke patients usually requires surgery to relieve intracranial pressure caused by bleeding. Surgical treatment for hemorrhagic stroke caused by an aneurysm or defective blood vessel can prevent additional strokes. Surgery may be performed to seal off the defective blood vessel and redirect blood flow to other vessels that supply blood to the same region of the brain.

Recently start endovascular treatment involves inserting a long, thin, flexible tube (catheter) into a major artery, usually in the thigh, guiding it to the aneurysm or the defective blood vessel and inserting tiny platinum coils (called stents) into the blood vessel through the catheter. Stents support the blood vessel to prevent further damage and additional strokes. Both of endovascular \& neurosurgical procedure need special care by anaesthesiologists. $10-30 \%$ stroke patients need admission in intensive care unit and the Anaesthesiologists have to play a vital role in the initial resuscitation, controlling of raised intracranial pressure, cardiovascular \& respiratory support or post operative care.

Acute care neurosurgery covers a broad range of disorders and procedures all sharing in common an emergent or urgent nature. This type of service requires 24-7 coverage by a team, comprising of neurosurgeons and neuroanaesthesiologists. A significant proportion (80-90\%) of neurosurgical emergencies were related to trauma only ten years back. Now there is change in the trends and more than 50 percent of emergency and urgent neurosurgical consultations involve a broad scope of brain and spinal disorders such as intracranial 
hemorrhages (bleeding within the brain), ventriculoperitoneal shunt, hemorrhagic in the spine, cerebral aneurysm ruptures, hemorrhagic strokes and sometime ischemic strokes. Primary intracerebral hemorrhage (ICH) accounts for $10 \%$ to $20 \%$ of strokes but carries the highest rates of mortality and morbidity of all stroke subtypes.

Most of the cerebrovascular diseases both hemorrhagic \& ischemic strokes were being managed conservatively even in ten years back. But during the last few years the practices are changing and the emergency neuro surgical procedures which need special attention by anaesthesiologists are gradually increasing. This increased surgical treatment of stroke patient not only involves the anaesthesiologist tasks in the operation theatre but there is increasing need of the anaesthesiologist expert involvement in the pre-opeartive management and in the ICU. Hence management of these group of patients is a new burden and challenges for the Anaesthesiologist.

There is increase trends of burden of emergency neuroanaesthesia for stroke patient are favourable in several developed areas of the world, but there are major problem in developing country like Bangladesh both from shortage of skill manpower with adequate facility. It is very much essential to increase the number of skilled neuroanaesthetist for providing emergency and acute care neurosurgical services for stroke patient. Though there is lack of interest for providing emergency neuroanaesthesia by the anaesthesiologist due to high mortality of hemorrhagic stroke patient.

The issue need proper addressing and policy have to be made to encourage the anaesthesiologist to become a skilled neuroanaesthesiologist.
Neuroanaesthesia subspeciality is now a demand of time.

(JBSA 2014; 27(1): 1-2)

Prof. M. Abdur Rahman

Department of Anaesthesia, Pain, Palliative \& Intensive Care Medicine

Dhaka Medical College

Dhaka, Bangladesh

\section{References}

1. Lewis B. Morgenstern, J. Claude Hemphill III, Craig Anderson and et all : AHA/ASA Guideline: Guidelines for the Management of Spontaneous Intracerebral Hemorrhage-A Guideline for Healthcare Professionals From the American Heart Association/American Stroke Association

2. Randell T, Niemelä M, Kyttä J, Tanskanen P, Principles of neuroanesthesia in aneurysmal subarachnoid hemorrhage: The Helsinki experience.

3. F Levi, F Lucchini, E Negri, and C La Vecchia, Trends in mortality from cardiovascular and cerebrovascular diseases in Europe and other areas of the world Heart. Aug 2002; 88(2): 119-124.

4. Morioka J, Fujii M, Kato S, Fujisawa H, Akimura T, Suzuki M, Kobayashi S; Japan Standard Stroke Registry Group (JSSR). Surgery for spontaneous intracerebral hemorrhage has greater remedial value than conservative therapy. Surg Neurol. 2006; 65: 67-72. 